\title{
Caracterização botânica-agronômica e do óleo essencial de acessos de manjericão
}

\section{Characterization botanical-agronomic and essential oil of basil accessions}

\author{
Henrique Guilhon de Castro ${ }^{*}$, Ronice Alves Veloso ${ }^{2}$, Dione Pereira Cardoso ${ }^{3}$, Aloísio Freitas Chagas Júnior ${ }^{4}$; Lillian \\ França Borges Chagas ${ }^{5}$
}

Resumo: Objetivou-se realizar a caracterização de quatro acessos e duas cultivares de manjericão por características fitoquímicas do óleo essencial e botânico-agronômicas em diferentes épocas de colheita. O experimento foi instalado utilizando-se um delineamento inteiramente casualizado em esquema de parcela subdivida, com quatro repetições. As parcelas consistiram de quatro acessos e duas cultivares de manjericão e as subparcelas por cinco épocas de amostragem. A análise de agrupamento por meio do método Tocher, utilizando as características botânico-agronômicas e fitoquímicas do óleo essencial, promoveu o agrupamento dos acessos silvestres no mesmo grupo evidenciando similaridade entre eles. Os acessos coletados na mesma região formaram grupo conjuntos. Observou-se variação na constituição dos grupos pelos acessos nas diferentes épocas de avaliação por características botânico-agronômicas. A divergência genética entre acessos e cultivares de manjericão, avaliada por características botânico-agronômicas, foi influenciada pelo estádio de desenvolvimento da espécie. A localização geográfica teve influência na caracterização e avaliação da divergência genética entre os acessos. Os constituintes majoritários presentes no óleo essencial das amostras avaliadas foram o linalol, nas cultivares, e o (E)- cinamato de metila, nos acessos silvestres.

Palavras-chave: Ocimum basilicum, recursos genéticos vegetais, plantas medicinais

\begin{abstract}
The objective of this study was to characterize four accessions and two basil cultivars by phytochemical characteristics of essential oil and botanical-agronomic in different harvest times. The experiment was installed in a completely randomized design in a subdivided plot scheme with four repetitions. The plots consisted of four accessions and two basil cultivars and the subplots for five sampling times. The grouping analysis by the method Tocher use botanical-agronomic and phytochemicals of essential oil traits, was efficient in the evaluation of genetic divergence between the accessions and cultivars of basil, as promoted the grouping of the wild accessions in the same group evidencing similarity between them. The accessions collected in the same region formed group together. Variation was observed for the group constitutions by accessions in the different sampling times by botanical-agronomical traits. The genetic divergence among basil accessions and cultivars, evaluated by botanical-agronomical traits, was influenced by development stage of specie. The geographical location influenced the characterization and evaluation of genetic divergence among accessions. The major constituents present in the essential oil of the samples were linalool, in cultivars, and $(E)$ - methyl cinnamate in wild accessions.
\end{abstract}

Key words: Ocimum basilicum, genetic plant resources, medicinal plants, essential oil.

\footnotetext{
*Autor para correspondência

Recebido para publicação em 20/03/2016; aprovado em 14/10/2016

${ }^{1}$ Doutor em Fitotecnia, Universidade Federal de Juiz de Fora, Juiz de Fora; 32-99995-0345, hguilhon@ mail.uft.edu.br

${ }^{2}$ Doutoranda em Produção Vegetal, Universidade Federal do Tocantins, Gurupi; ronice@uft.edu.br.

${ }^{3}$ Doutora em Fitotecnia, Universidade Federal do Tocantins, cardoso.dione@gmail.com

${ }^{4}$ Doutor em Biotecnologia, Universidade Federal do Tocantins, chagasjraf@bol.com.br

${ }^{5}$ Doutora em Produção vegetal, Universidade Federal do Tocantins, lillianfbc@uft.edu.br
} 


\section{INTRODUÇÃO}

A existência de variabilidade genética é condição fundamental para a evolução das espécies. A variabilidade genética é a base para a resistência à pressão do meio ambiente consistindo em matéria-prima para a seleção natural, a qual promove variações na constituição genética e atividade fisiológica entre populações e, consequentemente, entre espécies. Portanto, para determinado grau de pressão ambiental, indivíduos de mesma espécie apresentam respostas adaptativas diferentes (CASTRO et al., 2005; CABRAL et al., 2002).

Estudos acerca da divergência genética são importantes em programas de melhoramento, por fornecerem parâmetros para identificação dos genitores que, ao serem cruzados, possibilitam maior efeito heterótico na progênie e maior probabilidade de recuperar genótipos superiores nas gerações segregantes. A avaliação da divergência genética também é de grande valia no estudo da evolução das espécies, uma vez que provê informações a respeito dos recursos disponíveis e auxilia na localização e no intercâmbio de tais recursos (ARRIEL et al., 2007; CASTRO et al., 2011).

A erosão genética, que é a perda de recursos genéticos, têm como causas o aumento da população, a extensão da fronteira agrícola, a adoção de germoplasma elite responsável pela uniformização genética dos cultivos e a destruição dos centros de variabilidade genética. Essa perda de recursos fitogenéticos coloca em evidência a necessidade da conservação e caracterização das espécies (CASTRO et al., 2005; BOTREL et al., 2006; CASTRO et al., 2011).

$\mathrm{O}$ manjericão (Ocimum basilicum L.), pertencente à família Lamiaceae, é uma planta herbácea originária do Sudoeste Asiático, utilizada como planta medicinal e aromática, apresentando substâncias de interesse para as indústrias alimentícia, farmacêutica e cosmética. O manjericão é empregado na indústria culinária, de fitoterápicos e na medicina tradicional devido ao teor e composição do seu óleo essencial (SILVA et al., 2005; ÖZCAN; CHALCHAT, 2002; HANIF et al., 2011; BLANK et al., 2010; ROSADO et al., 2011; OLIVEIRA et al., 2013; SILVA et al., 2003; NASCIMENTO et al., 2011).

O estudo de aspectos agronômicos e do óleo essencial de acessos de Ocimum sp. é importante para a caracterização dos recursos fitogenéticos dessas espécies, permitindo a seleção de acessos promissores para o cultivo por apresentarem características superiores, e em relação ao teor de linalol no óleo essencial (BLANK et al., 2004; CASTRO et al., 2004; OTTAI et al., 2012). Conhecer as características da planta de interesse e do ambiente de cultivo contribui para a seleção de genótipos mais adaptados a ambientes específicos (CAMÊLO et al., 2011; KAMADA et al., 1999).

O objetivo deste trabalho foi realizar a caracterização de quatro acessos e duas cultivares de manjericão por características fitoquímicas do óleo essencial e botânicoagronômicas em diferentes épocas de colheita, coletados em diferentes regiões geográficas e cultivados nas mesmas condições.

\section{MATERIAL E MÉTODOS}

Neste trabalho foram estudados quatro acessos silvestres de manjericão e duas cultivares comerciais. Os acessos silvestres de manjericão $(O$. basilicum) foram coletados no

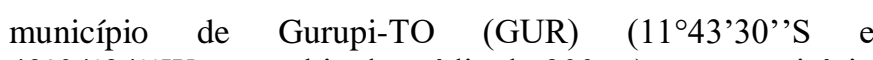
4904'34' $\mathrm{W}$, com altitude média de $300 \mathrm{~m}$ ) e no município de Monte Alegre-GO. No município de Monte Alegre foram coletados três acessos nas seguintes localidades: bairro Central (MAL) (1315'24'’S e 46 53'24'’W, com altitude média de $543 \mathrm{~m})$, bairro Montes Verdes (MVE) $\left(13^{\circ} 15^{\prime} 36^{\prime}\right.$ 'S e 46 $52^{\prime} 53^{\prime}$ 'W, com altitude média de $548 \mathrm{~m}$ ) e na Fazenda

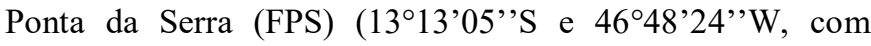
altitude média de $577 \mathrm{~m})$. As sementes da variedade comercial denominada manjericão roxo (MR) foram adquiridas no comércio local do município de Gurupi-TO e as da variedade Maria Bonita (MB) foram fornecidas pelo Horto de Plantas Medicinais da Universidade Federal de Lavras.

As exsicatas dos acessos coletados foram depositadas no herbário da Universidade Federal de Lavras com os números: MAL - 26.599; GUR - 26.603; FPS - 26.602; MVE - 26.601; MB - 26.920; e MR - 26.600.

O experimento foi conduzido em casa de vegetação na Universidade Federal do Tocantins, campus de Gurupi

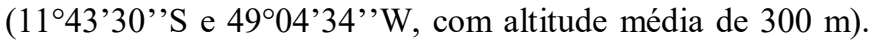
Utilizou-se o delineamento experimental inteiramente casualizado, em esquema de parcela subdividida, com quatro repetições. As parcelas foram constituídas por quatro acessos e duas cultivares de manjericão e as subparcelas por cinco épocas de amostragem com intervalos regulares de quinze dias. A unidade experimental foi constituída por dois vasos e em cada vaso duas plantas, totalizando 24 plantas por repetição.

As sementes de manjericão foram plantadas em vasos de polietileno preto com capacidade de dez litros, utilizando-se como substrato solo de barranco mais esterco bovino na proporção 2:1.

A análise química do substrato utilizado no experimento apresentou a seguinte composição química: $\mathrm{pH}\left(\mathrm{H}_{2} \mathrm{O}\right)=7,6$; $\mathrm{H}^{+}+\mathrm{Al}^{3+}=0,99 \mathrm{cmol}_{\mathrm{c}} \mathrm{dm}^{-3} ; \mathrm{Ca}^{2+}=2,73 \mathrm{cmol}_{\mathrm{c}} \mathrm{dm}^{-3} ; \mathrm{Mg}^{2+}=$ $2,14 \mathrm{cmol}_{\mathrm{c}} \mathrm{dm}^{-3} ; \mathrm{P}\left(\right.$ extrator Melich $\left.{ }^{1}\right)=107,4 \mathrm{mg} \mathrm{dm}^{-3} ; \mathrm{K}^{+}=$ $798,4 \mathrm{mg} \mathrm{dm}^{-3}$; matéria orgânica $=66,64 \mathrm{~g} \mathrm{dm}^{-3} ; \mathrm{CTC}=6,92$ cmolc $\mathrm{dm}^{-3}$ e $\mathrm{V} \%=87,48 \%$.

As avaliações das características foram iniciadas aos 42 dias após o plantio. As seguintes características foram avaliadas: altura das plantas, diâmetro do caule (a cinco centímetros do solo), número de inflorescências e vigor das plantas.

$\mathrm{Na}$ última época de avaliação, aos 102 dias após o plantio, as plantas foram coletadas realizando-se o corte rente ao solo e determinadas as seguintes características: massa fresca total, massa fresca das folhas, massa fresca das inflorescências, massa fresca dos ramos, número de folhas, número de inflorescências, número de ramos, massa seca das folhas, massa seca das inflorescências e massa seca dos ramos.

O óleo essencial das plantas de manjericão foi obtido por hidrodestilação a partir de amostras da parte aérea de plantas de manjericão desidratadas $(0,02 \mathrm{~kg})$ por um período de duas horas, com quatro repetições. As amostras foram colocadas em balão de fundo redondo contendo $1 \mathrm{~L}$ de água destilada, acoplado ao aparelho tipo Clevenger. O óleo essencial foi extraído da fase aquosa, utilizando-se pentano (4 x $40 \mathrm{~mL}$ ). As frações orgânicas obtidas foram reunidas e secadas com sulfato de magnésio anidro, filtradas e o solvente removido sob pressão reduzida em evaporador rotativo a $40^{\circ} \mathrm{C}$, em equipamento QUIMIS (modelo Q344B2). 
A identificação dos compostos do óleo essencial foi realizada por cromatografia em fase gasosa e espectrometria de massas (CG/EM) em equipamento Shimadzu, modelo QP 2010 Plus, com tempo de análise de 70 minutos, em coluna Equily-5 (30 m x 0,25 mm x 0,25 $\mu \mathrm{m})$. Foi utilizado hélio como gás de arraste com a velocidade de $1,8 \mathrm{~mL}$ min- 1 . As temperaturas do injetor e do detector foram $220^{\circ} \mathrm{C}$ e $240^{\circ} \mathrm{C}$, respectivamente. A temperatura inicial do forno foi de $60^{\circ} \mathrm{C}$ por $2 \mathrm{~min}$, seguido de um incremento de $3^{\circ} \mathrm{C}$ por $1 \mathrm{~min}$ até atingir $240^{\circ} \mathrm{C}$, sendo mantida constante por 15 minutos.

A identificação dos constituintes químicos foi feita por comparação dos espectros de massas com os espectros de massas disponíveis no banco de dados do equipamento, com a literatura e pelo índice aritmético de retenção relativo (IR) à série de alcanos C9-C22 (ADAMS, 2007).

Nas características botânico-agronômicas foram realizadas cinco análises de agrupamento em cada época de amostragem. Para cálculo da distância genética foi obtida a distância generalizada de Mahalanobis e para delimitação dos grupos de dissimilaridade dos acessos foi adotado o Método de Tocher. Nas características fitoquímicas do óleo essencial também foi utilizado o método de Tocher para delimitação dos grupos de dissimilaridade e no cálculo da distância genética foi utilizada a distância Euclidiana Média.

Para estimar a distância média dentro do grupo foi calculou-se a média das distâncias entre pares de acessos de um mesmo grupo; a distância entre grupos foi estimada pela média das distâncias entre todos os pares de acessos dos grupos. Utilizou-se o critério de Singh (1981) para identificar a contribuição relativa de cada característica para a diversidade entre os acessos (CASTRO et al., 2011).

A correlação de Pearson foi estimada entre as médias das características botânico-agronômicas (massa fresca total MFT, massa seca total - MST, massa fresca das folhas MFF, massa seca das folhas - MSF, massa seca das inflorescências - MSI e massa fresca das inflorescências MFI) e fitoquímicas do óleo essencial (metil cinamato - CIN, linalol - LIN e teor do óleo essencial - TO). As análises estatísticas foram realizadas no programa SAEG (RIBEIRO JÚNIOR \& MELO, 2009).

\section{RESULTADOS E DISCUSSÃO}

\section{Divergência genética por características botânico- agronômicas}

Observou-se que houve variação na composição dos grupos formados nas épocas de avaliação com base nas características botânico-agronômicas. Na segunda, terceira e quarta épocas de avaliação foram formados três grupos e na primeira e na quinta época dois grupos. Os acessos silvestres (MAL, GUR, FPS e MVE) mantiveram-se agrupados no mesmo grupo na primeira e na quinta época. O acesso GUR que na primeira e na última época de avaliação formava grupo com os outros acessos silvestres passou na segunda e terceira época de avaliação a constituir um grupo isolado dos outros acessos. $\mathrm{O}$ acesso FPS passou a formar um grupo isolado na quarta época de avaliação aos 87 dias após o plantio. As cultivares MB e MR ficaram agrupadas no mesmo grupo em todas as épocas de avaliação (Tabela 1).
Tabela 1. Grupos de similaridade entre quatro acessos e duas cultivares de manjericão (Ocimum basilicum) estabelecidos pelo Método de Tocher por meio de características botânicoagronômicas, em cinco épocas de avaliação. Gurupi.

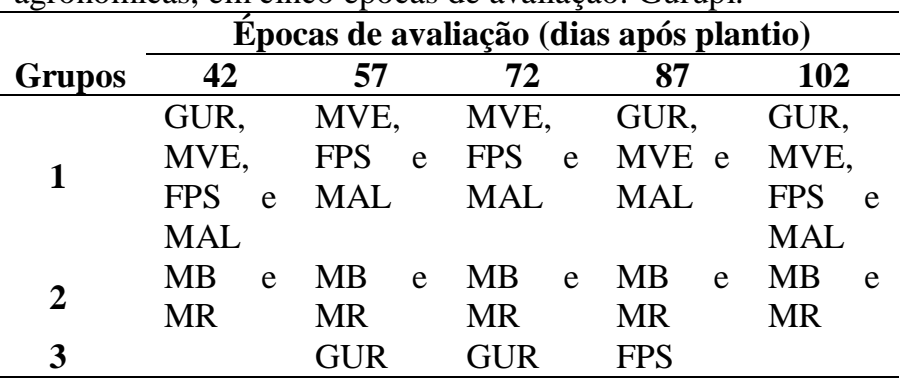

A característica que mais contribuiu para a divergência genética entre os acessos e cultivares foi o número de inflorescências (NI), que se destacou em todas as épocas de avaliação, exceto na última época em que a variável número de folhas (NF) apresentou maior contribuição à divergência genética. As características MFT, MFF, MFI, MFR, MSF e MST apresentaram contribuição relativa percentual para a divergência genética nula em todas as épocas de avaliação (Tabela 2).

Tabela 2. Contribuição relativa percentual para a divergência genética $\left(\mathrm{D}^{2}\right)$, das variáveis: altura (ALT), diâmetro do caule (DC), vigor (VIG), número de inflorescência (NI), número de folhas (NF), número de ramos (NR), massa seca das inflorescências (MSI) e massa seca dos ramos (MSR), de quatro acessos e duas cultivares de manjericão (Ocimum basilicum), em cinco épocas de avaliação. Gurupi

\begin{tabular}{llllll}
\hline & \multicolumn{5}{c}{ Épocas de avaliação } \\
\cline { 2 - 6 } Variáveis & $\mathbf{4 2}$ & $\mathbf{5 7}$ & $\mathbf{7 2}$ & $\mathbf{8 7}$ & $\mathbf{1 0 2}$ \\
\hline ALT & 26,1 & 33,3 & 20 & 20 & 0 \\
DC & 20 & 13,3 & 20 & 13,3 & 6,67 \\
VIG & 6,67 & 0 & 6,67 & 13,3 & 6,67 \\
NI & 46,7 & 53,3 & 53,3 & 53,3 & 20 \\
NF & - & - & - & - & 26,7 \\
NR & - & - & - & - & 20 \\
MSTI & - & - & - & - & 13,3 \\
MSTR & - & - & - & - & 6,67 \\
\hline
\end{tabular}

Outras observações de campo que destacaram a similaridade entre as cultivares MB e MR e entre os acessos silvestres por características botânico-agronômicas foi $\mathrm{o}$ tamanho das folhas e a coloração das flores. Nas cultivares MB e MR observou-se que o tamanho das folhas era maior que dos acessos silvestres com largura média de $2,45 \mathrm{~cm}$ e comprimento médio de $4,85 \mathrm{~cm}$. Nos acessos silvestres a largura média das folhas e o comprimento médio das folhas foram de $1,2 \mathrm{~cm}$ e $2,33 \mathrm{~cm}$, respectivamente. Nos acessos silvestres observou-se coloração branca das flores em todos os acessos (MAL, GUR, FPS e MVE), reforçando a similaridade entre os mesmos.

Castro et al. (2004) estudaram a divergência genética entre acessos de mentrasto com base em características botânico-agronômicas e concluíram que as características que mais contribuíram para a divergência genética entre acessos de mentrasto foi biomassa fresca, biomassa seca e número de sementes por inflorescência e as características que menos contribuíram foi altura e número de inflorescência por planta.

Diversos outros trabalhos sobre divergência genética com base em características botânico-agronômicas foram 
realizados, demonstrando o seu potencial para estudar a divergência genética em diferentes espécies de plantas medicinais (CASTRO et al., 2005; MARIOT et al., 2009; CASTRO et al., 2011; BOLINA et al., 2013; DARVISHZADEH et al., 2013; MEZETTE et al., 2013).

$\mathrm{Na}$ Tabela 3 são mostradas as distâncias de Mahalanobis $\left(\mathrm{D}^{2}\right)$ intra e intergrupos entre os seis acessos de manjericão nas cinco épocas de avaliação. Comparando os grupos I e II, a distância média intergrupo foi superior na última época de avaliação. No entanto, comparando os grupos II e III a distância média intergrupo foi maior na terceira época de avaliação. Este resultado demonstra a maior capacidade de distinção das características nessas épocas de amostragem.

Tabela 3. Distância de Mahalanobis $\left(D^{2}\right)$ intra e intergrupos entre quatro acessos e duas cultivares de manjericão (Ocimum basilicum), em cinco épocas de avaliação. Gurupi

\begin{tabular}{llll}
\hline Grupos & I & II & III \\
\hline I & $1,72(1)$ & 1,99 & - \\
& $4,30(2)$ & 8,13 & 4,32 \\
& $0,40(3)$ & 10,33 & 5,67 \\
& $0,49(4)$ & 10,93 & 2,26 \\
& $23,42(5)$ & 17,93 & - \\
\hline II & 1,86 & - \\
& & 2,02 & 13,85 \\
& & 1,24 & 27,81 \\
& & 1,19 & 6,92 \\
& & 128,29 & - \\
\hline
\end{tabular}

(1) = avaliação realizada aos 42 dias após plantio; (2) = avaliação realizada aos 57 dias após plantio; (3) = avaliação realizada aos 72 dias após plantio; (4) = avaliação realizada aos 87 dias após plantio; (5) = avaliação realizada aos 102 dias após plantio.

\section{Divergência genética por características fitoquímicas}

Os constituintes identificados no óleo essencial dos quatro acessos e das duas variedades do manjericão podem ser divididos em compostos terpenos e fenilpropanóides.

Os compostos terpenos identificados nos acessos e cultivares de manjericão com as concentrações relativas (\% área) foram os seguintes: $\alpha$-Pineno (MB- 0,22); Sabineno (MB- 0,37); $\beta$ - Pineno (MB- 0,77); $\beta$ - Mirceno $(0,43)$; Limoneno (MAL- 0,09, FPS- 0,23 e MB- 0,30); $\beta$-ocimeno (MB- 0,48); 1,8-cineol (MB- 13,09 e MR- 2,63); Hidrato de sabineno (MB- 0,2 e MR- 0,18); Óxido de linalol (MB- 0,07 e MR- 0,07); Fenchona (MR- 0,47); Linalol (MAL- 0,22, GUR- 0,28, FPS- 0,12, MVE- 4,43, MB- 36,32 e MR- 47,76); Cânfora (MB- 0,89 e MR- 1,13); Nerol (MB- 0,80 e MR$0,58)$; $\alpha$-cubebeno (MAL- 0,09); $\alpha$-copaeno (MAL- 0,09, GUR- 0,01, FPS- 0,04, MVE- 0,11, MB- 0,04); $\beta$-Elemeno (MR- 2,88); E-cariofileno (MAL- 5,18, GUR- 1,33, FPS2,66, MVE- 6,72, MB- 0,49 e MR- 1,21); $\alpha$-bergamoteno (MAL- 5,29, GUR- 1,13, FPS- 2,65, MVE- 7,66, MB- 5,09 e MR- 0,96); $\alpha$-Guaieno (MR- 0,69); (E)- $\beta$-Farneseno (MAL0,23, FPS- 0,11, MVE- 0,33); Germacreno D (MAL- 0,12, FPS- 0,05, MVE- 0,15, MB- 1,06 e MR- 1,14); $(E, E)-\alpha-$ Farneseno (MAL- 1,19, GUR- 0,31, FPS- 0,45 e MVE- 2,73); (E)- $\beta$-Guaieno (MB- 1,15 e MR- 1,14$)$; $\beta$-bisaboleno (MAL0,11 e MVE- 0,20); $\delta$-Amorfeno (MB- 0,75); $\gamma$-cadineno (MAL- 0,76, GUR- 0,14, FPS- 0,28 e MVE- 1,19); Estragol (MVE- 0,16 e MB- 28,41); Elemol (MB- 0,19); Nerolidol (MVE- 0,38, MB- 0,06 e MR- 0,15); Espatulenol (MR- 0,49); Óxido de cariofileno (MAL- 0,15, GUR- 0,04, MVE- 0,88,
MB- 0,05 e MR- 0,27); Veridiflorol (MVE- 0,34); Eudesmol 10-epi-gama (MB- 0,05); Epi- $\alpha$-Cadinol (MB- 2,19 e MR$3,14) ; \beta$-eudesmol (MB- 0,18 e MR- 0,33); e $\alpha$-cadinol (MVE- 0,26).

Em relação aos fenilpropanóides foram identificados os seguintes compostos: $(Z)$-cinamato de metila (MAL- 3,04, GUR- 2,46, FPS- 3,88, MVE- 24,15 e MR- 0,96); Eugenol (MR- 2,25); e $(E)$-Cinamato de metila (MAL- 80,93, GUR93,59, FPS- 88,65, MVE- 39,23, MB- 1,04 e MR- 22,22).

$\mathrm{O}$ constituinte majoritário presente no óleo essencial das amostras avaliadas nas cultivares MB e MR foi o linalol e nos acessos silvestres (MAL, GUR, FPS e MVE) o (E)cinamato de metila.

A análise de agrupamento com base nas características fitoquímicas do óleo essencial, definiu dois grupos. O primeiro grupo foi formado pelos acessos silvestres (GUR, FPS MAL e MVE) e a cultivar Manjericão Roxo e o segundo grupo apenas com a cultivar Maria Bonita. Este agrupamento se assemelha ao apresentado quando se avaliou a similaridade por meio das características botânico-agronômicas (Tabela 1), com exceção da cultivar Manjericão Roxo que ficou no mesmo grupo com os acessos silvestres no agrupamento realizado por características fitoquímicas.

Castro et al. (2011) avaliaram a divergência genética entre acessos de mentrasto (Ageratum conyzoides L.) e observaram que os acessos coletados na mesma região climática formaram grupo juntos. Este fato demonstra a importância da coleta de germoplasma em diferentes regiões climáticas para obtenção de maior representabilidade da variabilidade genética existente na espécie em estudo.

Os compostos químicos do óleo essencial que mais contribuíram para a divergência genética foram o $\alpha$-cubebeno e o $(E)$ - cinamato de metila $(33,3 \%)$. Em estudo realizado com mentrasto em Viçosa-MG, as características fitoquímicas que mais contribuíram para a divergência genética foram os compostos majoritários precoceno I e precoceno II (CASTRO et al., 2004).

As cultivares MB e MR estiveram no mesmo grupo na avaliação da divergência genética por características botânico-agronômicas e em grupos diferentes por características fitoquímicas. Por outro lado, nos acessos silvestres (MAL, GUR, FPS e MVE) observou-se tendência de formar grupo juntos com base nas características botânicoagronômicas e fitoquímicas. Apenas os acessos GUR e FPS, na análise de agrupamento por características botânicoagronômicas, formaram grupo isolado dos demais acessos silvestres na segunda, terceira e quarta época de amostragem.

Analisando a estrutura e composição dos grupos formados nas diferentes épocas de colheita pode-se observar variação na constituição dos grupos pelos acessos e cultivares. Esse fato demonstra a inconsistência da composição dos grupos formados nas diferentes épocas de colheita. Esses resultados também corroboram a importância da época de amostragem para estudos de divergência genética por características botânico-agronômicas, e sugerem que a divergência genética deve ser estimada em cada época de colheita, separadamente.

Verificou-se coeficiente de correlação não significativo entre o teor de $(E)$ - cinamato de metila e massa seca das inflorescências; entre teor de linalol e massa seca das folhas e massa fresca das inflorescências; e entre o teor de óleo essencial e massa seca das inflorescências. A maior produção de massa fresca total, massa seca total e massa 
fresca das folhas indicaram maior teor de linalol e teor de óleo essencial e menor teor de $(E)$-cinamato de metila (Tabela 4).

Tabela 4. Coeficiente de correlação de Pearson entre as características botânico-agronômicas (massa fresca totalMFT, massa seca total- MST, massa fresca das folhas- MFF, massa seca das folhas- MSF, massa seca das inflorescênciasMSI e massa fresca das inflorescências- MFI) e fitoquímicas $(\mathrm{CIN}=(E)$ - cinamato de metila, $\mathrm{LIN}=$ linalol e $\mathrm{TO}=$ teor do óleo essencial), de quatro acessos e duas cultivares de manjericão (Ocimum basilicum). Gurupi

\begin{tabular}{cccc}
\hline & CIN & LIN & TO \\
\hline MFT & $-0,8202 *$ & $0,7797^{*}$ & $0,9654^{* *}$ \\
MST & $-0,9006^{* *}$ & $0,7534^{*}$ & $0,9063^{* *}$ \\
MFF & $-0,8094^{*}$ & $0,7677^{*}$ & $0,9536^{* *}$ \\
MSF & $-0,8416^{*}$ & $0,6739^{\mathrm{ns}}$ & $0,8941^{* *}$ \\
MFI & $-0,9035^{* *}$ & $-0,5261^{\mathrm{ns}}$ & $0,9475^{* *}$ \\
MSI & $0,0551^{\mathrm{ns}}$ & $0,7751^{*}$ & $-0,4217^{\mathrm{ns}}$ \\
\hline
\end{tabular}

ns = não significativo pelo teste "t" a $5 \%$ de probabilidade; ${ }^{* *}=$ significativo pelo teste " $\mathrm{t}$ " a $1 \%$ de probabilidade; " = significativo pelo teste " $\mathrm{t}$ " a $5 \%$ de probabilidade.

\section{CONCLUSÕES}

As características botânico-agronômicas e fitoquímicas foram eficientes na caracterização de germoplasma e na avaliação da divergência genética entre os acessos e cultivares de manjericão. Deve também ser destacada a importância da coleta de germoplasma de manjericão em diferentes regiões geográficas com o objetivo de obter maior representatividade da variabilidade genética existente.

A localização geográfica teve influência na caracterização e avaliação da divergência genética entre os acessos e foi observado que o acesso coletado em Gurupi-TO (GUR) formou grupo sozinho na segunda e terceira época de avaliação. Por outro lado, os acessos coletados no estado de Goiás (MAL, FPS e MVE) formaram grupo juntos em todas as épocas de avaliação, com exceção da quarta época quando o acesso FPS formou grupo sozinho. Desta forma, o acesso GUR pode ser selecionado para orientar cruzamentos no programa de melhoramento genético de $O$. basilicum.

O constituinte majoritário presente no óleo essencial das amostras avaliadas nas cultivares MB e MR foi o linalol e nos acessos silvestres (MAL, GUR, FPS e MVE) o $(E)$ - cinamato de metila.

\section{AGRADECIMENTOS}

Ao Conselho Nacional de Desenvolvimento Científico e Tecnológico (CNPq) e a Coordenação de Aperfeiçoamento de Pessoal de Nível Superior (CAPES) pelas bolsas de pesquisa e apoio financeiro; à FAPEMIG por apoio financeiro.

Aos Professores Luiz Cláudio A. Barbosa e Mário C. Guerreiro pela colaboração na análise do óleo essencial.

\section{REFERÊNCIAS}

ADAMS, R. P. Identification of essential oil components by gas chromatography/mass spectroscopy. 4 Edition. Illinois USA: Allured Publishing Corporation, Carol Stream, 2007. 804 p.
ARRIEL, N. H. C.; MAURO, A. O. D.; ARRIEL, E. F.; UNÊDA-TREVISOLI, S. H.; COSTA, M. M.; BÁRBARO, I. M.; MUNIZ, F. R. S. 2007. Genetic divergence in sesame based on morphological and agronomic traits. Crop Breeding and Applied Biotechnology, v. 7, n. 3, p. 253-61, 2007.

BLANK, A. F.; CARVALHO FILHO, J. L. S.; SANTOS NETO, A. L.; ALVES, P. B.; ARRIGONI-BLANK, M. F.; SILVA-MANN, R.; MENDONÇA, M. C. Caracterização morfológica e agronômica de acessos de manjericão e alfavaca. Horticultura Brasileira, v. 22, n. 1, p. 113-116, 2004.

BLANK, A. F.; SOUZA, E. M.; PAULA, J. W. A.; ALVES, P. B. Comportamento fenotípico e genotípico de populações de manjericão. Horticultura Brasileira, v. 28, n. 3, p. 305-310, 2010 .

BOLINA, C. O.; MARCHESE, J. A.; CAPELIN, D.; BERTONCELLI, D. J.; PINNOW, C.; BENIN, G.; FOGLIO, M. A. Correlation among agronomic, physiological and phytochemical traits in Artemisia annua L. varieties. Revista Brasileira de Plantas Medicinais, v. 15, n. 1, p. 135-141, 2013.

BOTREL, M. C. G.; SOUZA, A. M.; CARVALHO, D.; PINTO, S. I. C.; MOURA, M. C. O.; ESTOPA, R. A. Caracterização genética de Calophyum brasiliense Camb. em duas populações de mata ciliar. Revista Árvore, v. 30, n. 5, p. 821-827, 2006

CABRAL, B. L. R.; SOUZA, J. A.; ANDO, A.; VEASEY, E. A.; CARDOSO, E. M. R. Isoenzymatic variability of cassava accessions from different regions in Brazil. Scientia Agrícola, v. 59, n. 3, p. 521-7, 2002.

CAMÊLO, L. C. A.; BLANK, A. F.; EHLERT, P. A. D.; CARVALHO, C. R. D.; ARRIGONI-BLANK, M. F.; MATTOS, J. Caracterização morfológica e agronômica de acessos de erva cidreira-brasileira [Lippia alba (Mill.) N. E. Br.]. Scientia Plena, v. 7, n. 5, p. 1-8, 2011.

CASTRO, H. G.; SANTOS, G. R.; MOMENTÉ, V. G.; SILVA, D. J. H.; RIBEIRO JÚNIOR, J. I. Estudo da divergência genética por características morfológicas entre acessos de mentrasto (Ageratum conyzoides L.) coletados no Estado do Tocantins em diferentes épocas. Revista Brasileira de Plantas Medicinais, v. 13, n. 1, p. 24-29, 2011.

CASTRO, H. G.; SILVA, D. J. H.; OLIVEIRA, L. O.; FERREIRA, F. A.; SAKIYAMA, N. S.; RIBEIRO JÚNIOR, J. I. Stability of genetic divergence among five mentrasto accessions in two nvironments. Crop Breeding and Applied Biotechnology, v. 5, n. 3, p. 332-338, 2005.

CASTRO, H. G.; SILVA, D. J. H.; OLIVEIRA, L.O.; FERREIRA, F. A.; SAKIYAMA, N. S.; BARBOSA, L. C. A.; RIBEIRO JÚNIOR, J. I. Diversidade genética entre acessos de mentrasto avaliada por características botânicoagronômicas, moleculares e fitoquímicas. Revista Ceres, v. 51, n. 294, p. 227-241, 2004.

DARVISHZADEH, R.; MIRZAEI, L.; MALEKI, H. H.; LAURENTIN, H.; ALAVI, S. R. Genetic variation in oriental tobacco (Nicotiana tabacum L.) by agro-morphological traits 
and simple sequence repeat markers. Revista Ciência Agronômica, v. 44, n. 2, p. 347-355, 2013.

HANIF, M. A.; AL-MASKARI, M. Y.; AL-MASKARI, A.; AL-SHUKAILI, A.; AL-MASKARI, A. Y.; AL-SABAHI, J. N. Essential oil composition, antimicrobial and antioxidant activities of unexplored Omani basil. Journal of Medicinal Plants Research, v. 5, n. 5, p. 751-757, 2011.

KAMADA, T.; CASALI, V. W. D.; BARBOSA, L. C. A.; FORTES, I. C. P.; FINGER, F. L. Plasticidade fenotípica do óleo essencial em acessos de manjericão (Ocimum basilicum L.). Revista Brasileira de Plantas Medicinais, v. 1, n. 2, p. 1322, 1999.

MARIOT, M. P.; BARBIERI, R. L.; CORRÊA, F.; BENTO, L. H. G. Variabilidade genética para caracteres morfológicos e fisiológicos em espinheira-santa (Maytenus ilicifolia (Schrad.) Planch. e M. aquifolium Mart.). Revista Brasileira de Plantas Medicinais, v. 11, n. 3, p. 310-316, 2009.

MEZETTE, T. F.; BLUMER, C. G.; VERSEY, E. A. Morphological and molecular diversity among cassava genotypes. Pesquisa Agropecuária Brasileira, v. 48, n. 5, p. 510-518, 2013.

NASCIMENTO, J.; BARBOSA, L. C. A.; PAULA, V. F.; DAVID, J. M.; FONTANA, R.; SILVA, L. A. M.; FRANÇA, R. S. Chemical composition and antimicrobial activity of essential oils of Ocimum canum Sims. and Ocimum selloi Benth. Anais da Academia Brasileira de Ciências, v. 83, n. 83, p. 787-799, 2011.

OLIVEIRA, R. A.; MOREIRA, I. S.; OLIVEIRA, F. F. Linalool and methyl chavicol present basil (Ocimum sp.) cultivated in Brazil. Revista Brasileira de Plantas Medicinais, v. 15, n. 2, p. 309-311, 2013.
OTTAI, M. E. S.; SAYEDA, S. A.; EL DIN, M. M. Genetic Variability Among Some Quantitative Characters, Insecticidal Activity and Essential Oil Composition of Two Egyptian and French Sweet Basil Varieties. Australian Journal of Basic and Applied Sciences, v. 6, n. 3, p. 185-192, 2012.

ÖZCAN, M.; CHALCHAT, J. C. Essential oil composition of Ocimum basilicum L. and Ocimum minimum L. in Turkey. Czech Journal of Food Sciences, v. 20, n. 6, p. 223-228, 2002.

RIBEIRO JÚNIOR, J. I.; MELO, A. L. P. Guia prático para utilização do SAEG. Viçosa: UFV, 2009. 287p.

ROSADO, L. D. S.; PINTO, J. E. B. P.; BOTREL, P. P.; BERTOLUCCI, S. K. V.; NICULAU, E. S.; ALVES, P. B. Influência do processamento da folha e tipo de secagem no teor e composição química do óleo essencial de manjericão cv. Maria Bonita. Ciência e Agrotecnologia, v. 35, n. 2, p. 291-296, 2011.

SILVA, F.; SANTOS, R. H. S.; ANDRADE, N. J.; BARBOSA, L. C. A.; CASALI, V. W. D.; LIMA, R. R.; PASSARINHO, R. V. M. Basil conservation affected by cropping season, harvest time and storage period. Pesquisa Agropecuária Brasileira, v. 40, n. 4, p. 323-328, 2005.

SILVA, F.; SANTOS, R. H. S.; DINIZ, E. R.; BARBOSA, L. C. A.; CASALI, V. W. D.; Lima, R. R. Teor e composição do óleo essencial de manjericão (Ocimum basilicum L.) em dois horários e duas épocas de colheita. Revista Brasileira de Plantas Medicinais, v. 6, n. 1, p. 33-38, 2003.

SINGH, D. The relative importance of characters affecting genetic divergence. Indian Journal Genetic and Plant Breeding, v. 41, p. 237-245, 1981. 